\title{
INFLUENCIA DE CUBIERTAS VEGETALES EN LA DISPONIBILIDAD DE AGUA Y RENDIMIENTO EN VIÑEDOS DE SECANO
}

\section{EFFECT OF GRASS COVER CROPS ON WATER AVAILABILITY AND YIELD IN NON-IRRIGATED VINEYARDS}

\author{
Celerino Quezada ${ }^{1 *}$, Marco Sandoval $^{1}$, Carlos Ovalle $^{2}$, Víctor Pérez $^{1}$ \\ ${ }^{1}$ Universidad de Concepción, Facultad de Agronomía, Casilla 537, Chillán, Chile. \\ ${ }^{2}$ Instituto de Investigaciones Agropecuarias, CRI Quilamapu, Casilla 426, Chillán, Chile. \\ *Autor para correspondencia E-mail: cequezad@udec.cl
}

\section{RESUMEN}

El uso de cubiertas vegetales en viñedos de secano es una alternativa sustentable en suelos delgados, de laderas y con baja capacidad de retención de humedad. Se realizó un estudio en una zona mediterránea del centro sur de Chile para evaluar el efecto de diferentes cubiertas vegetales sembradas en la entrehilera sobre el contenido de agua en el suelo y rendimiento de un viñedo de secano Vitis vinifera L. 'Cabernet Sauvignon'. El diseño experimental fue de bloques completos al azar con cinco tratamientos y cuatro repeticiones. Los tratamientos consistieron en diferentes combinaciones de gramíneas y leguminosas y un testigo sin vegetación obtenido con labranza. Las evaluaciones realizadas fueron: contenido volumétrico de agua, agua aprovechable, rendimiento del viñedo y vigor de la vid. Los resultados obtenidos indican que las coberturas vegetales no afectaron la disponibilidad de agua para la planta, ni comprometieron el rendimiento de la vid. La mejor combinación de especies fue festuca con trébol subterráneo y trébol balansa. vid.

Palabras clave: vid, Vitis vinifera, viña, entrehilera, disponibilidad de agua, mezclas forrajeras, vigor

\begin{abstract}
The use of cover crops in non-irrigated (rainfed) vineyards is a sustainable alternative in shallow hillside and low water retention soils. A study was conducted in the Mediterranean zone of centralsouthern Chile in order to evaluate the effect of different types of inter-row cover crops on soil water content and yield in a non-irrigated vineyard Vitis vinifera L. 'Cabernet Sauvignon'. The experiment was carried out using a randomized complete block design with five treatments and four replicates. The treatments consisted of different mixtures of grass and legume species and a control treatment (without vegetation cover). Soil volumetric water content, soil water availability, grape yield and vine vigor were evaluated. The results obtained showed that the cover crops evaluated did not affect soil water availability, grape yield or vine vigor. The mixture with the best performance was tall fescue, subterranean clover and balansa clover.
\end{abstract}

Key words: vine, Vitis vinifera, inter-row, grass mixtures, water availability, vine vigor.

Recibido: 13 marzo 2020. Aceptado: 12 mayo 2020. 


\section{INTRODUCCIÓN}

En las últimas décadas el uso de cubiertas vegetales ha sido una práctica de manejo habitual del piso del viñedo, debido a sus efectos benéficos en la protección del suelo contra erosión y escurrimiento superficial, mejoramiento de la estructura del suelo, capacidad de retención de agua, disminución de la evaporación del suelo y reducción en el consumo de agua (McGourty et al., 2008; Guerra y Steenwerth, 2012; Steenwerth et al., 2013; Medrano et al., 2015). Además, las cubiertas vegetales ayudan a regular la expresión vegetativa de la vid, mejorando la composición de la baya y reduciendo costos de manejo (TrigoCórdoba et al., 2015; Bouzas-Cid et al., 2018). Sin embargo, su desarrollo está limitado debido a un severa competencia por agua y nutrientes en viñedos de zonas mediterráneas (Celette et al., 2008; Pou et al., 2011; Guerra y Steenwerth, 2012).

Las cubiertas vegetales modifican la distribución espacial y temporal del agua en el perfil del suelo (Cellete et al., 2008). Steenwerth et al. (2016) determinaron diferencias significativas en el contenido de agua en volumen, pero sin afectar el rendimiento y composición de la fruta en 'Merlot' de 2 y 3 años. Respecto de su efecto en las propiedades físicas, Haruna y Nkongolo (2015) determinaron una disminución de la densidad aparente en 3,5\%. Wheaton et al. (2008), en suelos arcillosos y profundidad de $500 \mathrm{~mm}$, reportaron suelos menos compactados $(<1 \mathrm{MPa})$ y una mayor concentración de raíces del viñedo $5,05 \mathrm{~cm} \mathrm{~cm}^{-3}$ suelo en la profundidad entre $0 \mathrm{y}$ $100 \mathrm{~mm}$.

En la actualidad, en la Región del Maule, Chile, existen 52.617 ha de viñedos plantados en zonas de secano, en suelos de lomajes susceptibles a la erosión hídrica, pero que presentan un clima mediterráneo adecuado para producir vinos de calidad (Ruiz, 2020). La entrehilera del viñedo, por el tipo de suelo y las labores de manejo, presenta un alto grado de compactación con resistencia a la penetración de $5 \mathrm{MPa}$ (Quezada et al., 2014). Una de las alternativas para mejorar esta limitante, es el establecimiento de coberturas vegetales, porque las raíces profundizan en el suelo, mejoran la agregación de las partículas y producen gran cantidad de biomasa que ayuda a incrementar el contenido de materia orgánica (Pérez-Bermúdez et al., 2016). Las coberturas vegetales contribuyen a reducir la susceptibilidad del suelo a la compactación, en función de las especies que la componen, longitud del crecimiento y aporte de biomasa. El contenido de materia orgánica en los primeros $20 \mathrm{~cm}$ de un suelo granítico no se vio afectado por la inclusión de cubiertas vegetales entre las hileras de viñedos después de 2 años de realizada la siembra (Ovalle et al., 2007). Es importante la elección de las especies que conforman la cubierta vegetal, para que el ciclo de crecimiento no interfiera directamente con el crecimiento de la vid y además, que se regeneren en forma natural en los años siguientes a partir de semillas que se producen in situ, especialmente leguminosas que tienen efectos positivos en el suelo (Celette et al., 2005; McGourty et al., 2008; Ripoche et al., 2010). No hay claridad sobre las interacciones de la asociación de plantas perennes-herbáceas en el subsuelo y de sus efectos en el vigor de la vid y reducción del rendimiento (Cellete et al., 2008). En áreas mediterráneas, plantas de temprana senescencia y auto-resiembra natural, como Dactylis sp., Medicago sp. y Trifolium sp., pueden mejorar la cobertura del suelo y la competencia por el agua (Medrano et al., 2015).

La investigación de la competencia por el recurso agua entre viñedo y cubierta vegetal ha tenido resultados contradictorios, debido a diferentes condiciones de suelo y clima (TrigoCórdoba et al., 2015). Por los resultados dispares de las cubiertas vegetales, pocos agricultores están estableciendo esta tecnología en zonas semiáridas, porque sus ventajas son poco claras, ya que producen pérdidas de rendimiento en áreas con menos de $1.000 \mathrm{~mm}$ de lluvia anual (Medrano et al., 2015). Por su parte, Blanco-Canqui et al. (2015) determinaron que la competencia con el viñedo es la principal razón para no adoptar el intercultivo, por lo que se requiere de mayor investigación en zonas mediterráneas, para selección de especies, siembra, y técnicas de manejo. Esto permitiría lograr que los servicios ecosistémicos, como control de malezas, regulación de plagas y enfermedades, purificación del agua, transitabilidad y mantenimiento da la biodiversidad, según suelo y clima, no afecten la producción del viñedo y los retornos económicos de los agricultores (García et al., 2018).

Por lo anterior, el objetivo de esta investigación fue evaluar el efecto de diferentes combinaciones de gramíneas y leguminosas establecidas en la entrehilera sobre el contenido de agua, rendimiento y vigor de un viñedo de secano 'Cabernet Sauvignon' en un suelo Typic Palexeralfs en la zona centro sur de Chile.

\section{MATERIALES Y MÉTODOS}

\section{Sitio experimental}

El experimento se realizó en el predio La Estrella (35ํํㄱ' S; 72ำ13' O, 179 m.s.n.m.), comuna de Cauquenes, Región del Maule, Chile. El suelo pertenece a la Serie Cauquenes (fine, kaolinitic mesic, Typic Palexeralfs), originado a partir de 
formaciones graníticas, con topografía de lomajes, textura arcillosa a franco-arcillo-arenosa, y con baja infiltración de agua (Stolpe, 2006).El análisis granulométrico del suelo determinó $11,8 \%$ arcilla, $68,5 \%$ arena y $19,7 \%$ limo, y las constantes hídricas Capacidad de Campo $=13,96 \%$ hbss, Punto de Marchitez Permanente $=7,49 \%$ hbss, y densidad aparente de $1,45 \mathrm{~g} \mathrm{~cm}^{-3}$. El suelo presentó un $\mathrm{pH}$ (agua 1:2,5) 6,65, MO 1,55\%, N 1,93 $\mathrm{mg} \mathrm{kg}^{-1}, \mathrm{P} 5,47$ $\mathrm{mg} \mathrm{kg}^{-1}$ y K $207,1 \mathrm{mg} \mathrm{kg}^{-1}$

El clima es templado mediterráneo, con temperatura media anual de $14,7^{\circ} \mathrm{C}$, una mínima media del mes más frío (Julio) de $4,7^{\circ} \mathrm{C}$, y una máxima media del mes más cálido (enero) de $27^{\circ} \mathrm{C}$. El período libre de heladas varía entre 6 a 7 meses, desde octubre a abril, y las horas de frío suman 1.154. La precipitación media anual de $676 \mathrm{~mm}$ y la evapotranspiración potencial de $1.244 \mathrm{~mm}$. La estación seca es de 6 meses, desde noviembre hasta abril. La suma de temperaturas base $10^{\circ} \mathrm{C}$ es de 1.727 grados-día (Reyes y Mejías, 2011).

El viñedo es cultivar Cabernet Sauvignon, con 10 años de edad, conducido en espaldera de doble cruceta, con sistema de poda de cordón apitonado, 12 yemas por planta, y un marco de plantación de $1,0 \mathrm{~m}$ sobre la hilera y 3,5 m entrehilera (2.857 plantas ha-1) $^{-1}$.

\section{Cubiertas vegetales}

Las cubiertas vegetales fueron establecidas en mayo de 2005 y las evaluaciones físicas se realizaron entre octubre 2007 y febrero 2008. Las especies utilizadas fueron: pasto ovillo (Dactylis glomerata), trébol subterráneo (Trifolium subterraneum), trébol balansa (Trifolium michelanium), ballica inglesa (Lolium multiflorum), falaris (Phalaris aquatica) y festuca (Festuca arundinacea). La preparación del suelo se realizó mediante dos labores de arado cincel y una de rastra de discos. La siembra se efectuó con una máquina sembradora manual (Planet Jr. 300A, Allen \& Co., Philadelphia, Pennsylvania, USA), el 25 de mayo de 2005, en hileras separadas a $20 \mathrm{~cm}$ y dispuestas en el mismo sentido de la plantación de vid. La fertilización para el establecimiento consistió en una aplicación al voleo y posterior incorporación de $150 \mathrm{~kg} \mathrm{ha}^{-1}$ de $\mathrm{P}_{2} \mathrm{O}_{5}$ como superfosfato triple, $50 \mathrm{~kg} \mathrm{ha}^{-1}$ de N como urea, $500 \mathrm{~kg} \mathrm{ha}^{-1}$ de CaO como fertiyeso, 85 $\mathrm{kg} \mathrm{ha}^{-1}$ de $\mathrm{K}_{2} \mathrm{O}$ como muriato de potasio, $200 \mathrm{~kg}$ ha-1 de $\mathrm{S}$ como sulpomag, y $20 \mathrm{~kg} \mathrm{ha}^{-1}$ de B como boronatrocalcita. Todas las parcelas recibieron anualmente una fertilización de mantención de $50 \mathrm{~kg} \mathrm{ha}^{-1}$ de $\mathrm{P}_{2} \mathrm{O}_{5}$ como superfosfato triple.

Tabla 1. Dosis de semillas usadas en los tratamientos de cubiertas vegetales sembradas en la entrehilera de un viñedo de secano 'Cabernet Sauvignon' en un suelo Typic Palexeralf de la región centro sur de Chile.

Table 1. Seed doses used in the inter-row cover crop treatments sown in a 'Cabernet Sauvignon' nonirrigated vineyard in a Typic Palexeralf soil of central-southern Chile.

\begin{tabular}{|c|c|c|c|}
\hline Tratamiento & Especie & Cultivar & $\begin{array}{c}\text { Dosis semilla } \\
\quad\left(\mathrm{kg} \mathrm{ha}^{-1}\right)\end{array}$ \\
\hline $\mathrm{T} 1$ & Sin cubierta vegetal & & \\
\hline \multirow[t]{4}{*}{$\mathrm{T} 2$} & Dactylis glomerata & Currie & 12,0 \\
\hline & Trifolium subterraneum & Campeda & 9,0 \\
\hline & Trifolium subterraneum & Antas & 9,5 \\
\hline & Trifolium michelanium & Paradama & 0,9 \\
\hline \multirow[t]{4}{*}{ T3 } & Lolium multiflorum & Wimmera & 12,0 \\
\hline & Trifolium subterraneum & Campeda & 9,0 \\
\hline & Trifolium subterraneum & Antas & 9,5 \\
\hline & Trifolium michelanium & Paradana & 0,9 \\
\hline \multirow[t]{4}{*}{$\mathrm{T} 4$} & Phalaris aquatica & Holdfast & 10,0 \\
\hline & Trifoliujm subterraneum & Campeda & 9,0 \\
\hline & Trifolium subterraneum & Antas & 9,5 \\
\hline & Trifoluium michelanium & Paradana & 0,9 \\
\hline \multirow[t]{4}{*}{ T5 } & Festuca arundinacea & Manade & 12,0 \\
\hline & Trifolium subterraneum & Campeda & 9,0 \\
\hline & Trifolium subterraneum & Antas & 9,5 \\
\hline & Trifolium michelanium & Paradana & 0,9 \\
\hline
\end{tabular}




\section{Diseño experimental}

El diseño experimental fue de bloques completos al azar con cinco tratamientos y cuatro repeticiones. Cada tratamiento se implementó en parcelas de tres hileras de $25 \mathrm{~m}$ de largo por 3,5 $\mathrm{m}$ de ancho; no obstante, el ancho de la cubierta fue de $3 \mathrm{~m}$, dejando para ello $25 \mathrm{~cm}$ sin sembrar antes de la línea en la hilera de plantación con el objetivo de dejar libre de pasto alrededor y debajo de las plantas de vid. El área total del experimento fue de $5.250 \mathrm{~m}^{2}$. Los tratamientos investigados fueron los siguientes: (T1) testigo sin vegetación; (T2) pasto ovillo (Dactylis glomerata 'Currie') y leguminosas anuales; (T3) ballica anual (Lolium multiflorum 'Wimmera') y leguminosas anuales; (T4) falaris (Phalaris aquatica 'Holdfast') y leguminosas anuales; (T5) festuca (Festuca arundinacea 'Manade') y leguminosas anuales.

Las leguminosas anuales utilizadas en cada mezcla fueron trébol subterráneo (Trifolium subterraneum 'Campeda' y 'Antas') y trébol balansa (Trifolium michelanium 'Paradana'). Las especies fueron seleccionadas según investigaciones preliminares realizadas en el Centro Experimental Cauquenes del Instituto de Investigaciones Agropecuarias (INIA). Las combinaciones de especies y dosis de semilla para cada tratamiento se presentan en la Tabla 1. Las leguminosas se inocularon con el rizobio recomendado en dosis de $10 \mathrm{~g}$ de inóculo $\mathrm{kg}^{-1}$ de semilla.

\section{Evaluaciones \\ Contenido volumétrico de agua en el suelo}

El contenido volumétrico de agua en el suelo se midió semanalmente, desde el 16 de octubre de 2007 hasta el 26 de febrero de 2008, fechas que corresponden al periodo entre inicio de brotación y pinta en la zona, mediante una sonda de neutrones Hydroprobe 503 DR con fuente radiactiva Americio-241- Berilio (Campbell Pacific Nuclear, California, USA), a profundidades de 0-20, 20-40, 40-60, 60-80 y 80$100 \mathrm{~cm}$, entre y sobre hileras de plantación. Para esto se enterraron tubos de acceso de aluminio hasta 1,0 $\mathrm{m}$ de profundidad en la entrehilera y en la sobrehilera del viñedo para cada tratamiento y repetición (dos por parcela), con un total de 40 tubos en todo el ensayo. La instalación del tubo de acceso y la calibración del equipo se realizó según la metodología propuesta por Quezada et al. (2010). La curva de calibración del instrumento se obtuvo relacionando humedad volumétrica del suelo $(\theta)$ con conteo relativo (CR), obteniendo una ecuación $\theta=5,631 \mathrm{CR}+15,048\left(\mathrm{R}^{2}=0,89\right)$ mediante 32 mediciones entre límite superior e inferior de contenido de agua en el suelo.

\section{Propiedades físicas del suelo}

El muestreo del suelo se realizó a las profundidades de $0-5,5-10$ y $10-20 \mathrm{~cm}$, con barreno agrológico, en tres repeticiones por cada tratamiento. Las muestras fueron analizadas en el Laboratorio de Física de Suelos de la Facultad de Agronomía, Universidad de Concepción, Campus Chillán, determinando la textura por el método del hidrómetro, densidad aparente por el método del terrón, y retención de humedad por el método de la olla a presión (Sandoval et al., 2012).

\section{Rendimiento}

La cosecha se realizó en forma manual los días 6 y 7 de mayo del año 2008, muestreando 5 plantas por hilera por cada tratamiento y repetición, con un total de 300 plantas. La poda se hizo el 21 de agosto del 2008 en 5 plantas por hilera de un total de 24 plantas. El rendimiento del viñedo se evaluó mediante peso de uva ( $g$ planta $^{-1}$ ), peso de 10 bayas (g) y peso de poda ( $g$ planta $^{-1}$ ) El material colectado se pesó empleando balanzas digitales en el Laboratorio de Enología de la Estación Experimental INIA Cauquenes.

\section{Análisis estadístico.}

Los resultados de los parámetros físicos y de contenido volumétrico de agua en el suelo fueron sometidos a un análisis de varianza (ANDEVA) y para la comparación de medias se aplicó el test de Duncan, con un 95\% de confianza, utilizando el software InfoStat Profesional 2008 (Balzarini et al., 2008). Los valores en porcentaje fueron normalizados mediante la transformación $(x+$ $0,5)^{1 / 2}$.

\section{RESULTADOS Y DISCUSIÓN}

\section{Contenido volumétrico de agua en el suelo en la entrehilera}

Los valores de humedad volumétrica obtenidos en la entrehilera a la profundidad de $0-20 \mathrm{~cm}$ no presentaron diferencias estadísticas significativas (Fig. 1), pero el tratamiento de festuca con trébol subterráneo y trébol balansa (T5), presentó el mayor contenido de humedad durante toda la temporada, lo que es importante para una zona de secano. El resto de las combinaciones de especies mostraron contenido de humedad muy similar cercanos al $16 \%$.

Las profundidades de 20-40 y $40-60 \mathrm{~cm}$ presentaron un comportamiento similar en sus variaciones de humedad. A los 20-40 cm (Fig. 2) el tratamiento T5, con festuca y tréboles anuales, muestra el mayor valor $(18,41 \%)$, en cambio el tratamiento T2, con pasto ovillo, trébol subterráneo y trébol balansa, presentó el menor valor (16,57 \%). A 40-60 cm (Fig. 3) estos 


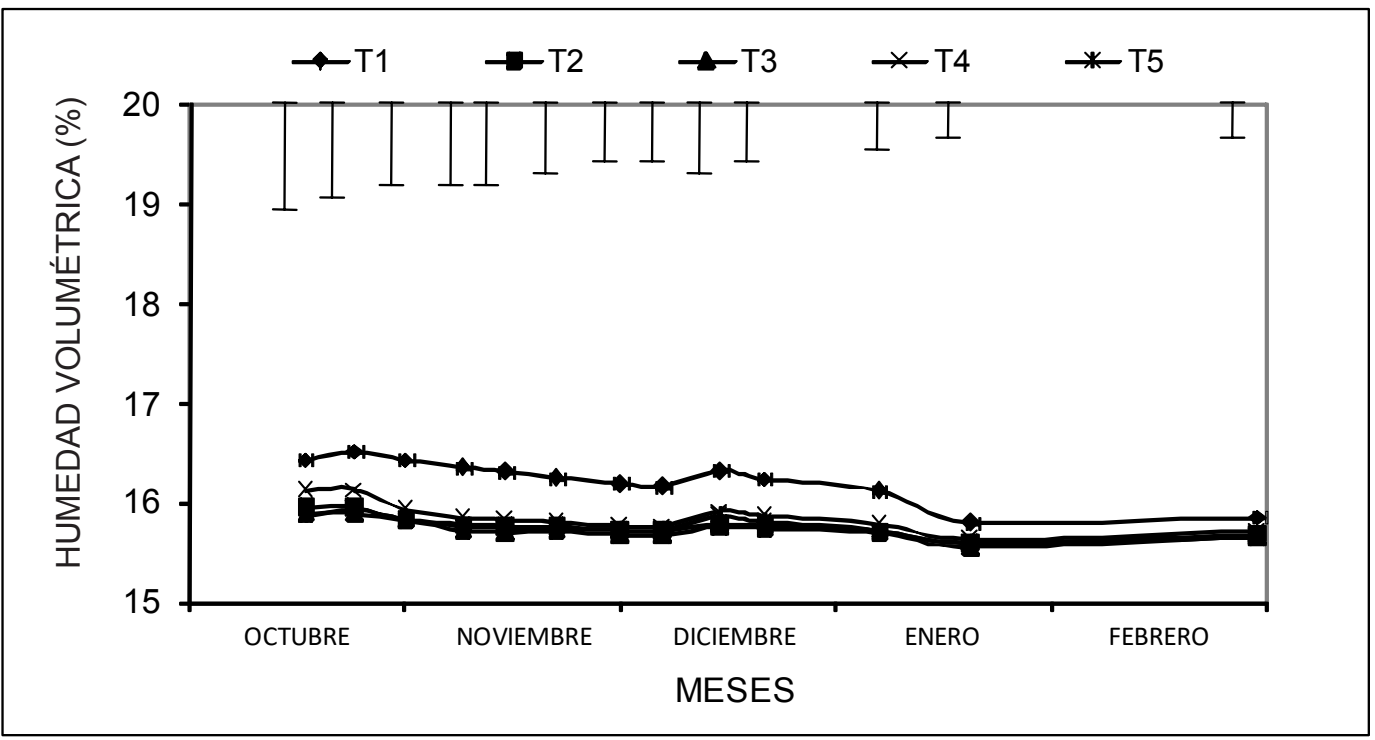

Fig. 1. Humedad volumétrica (\%) en un viñedo de secano Vitis vinifera 'Cabernet Sauvignon' en un suelo Typic Palexeralf a la profundidad de 0-20 cm bajo diferentes cubiertas vegetales entrehilera en la zona centro-sur de Chile.

Fig. 1. Soil volumetric water content $(\%)$ at $0-20 \mathrm{~cm}$ depth in a non-irrigated vineyard (Vitis vinifera 'Cabernet Sauvignon') under different inter-row cover crops in a Typic Palexeral soil of central-southern Chile.

T1: testigo, sin vegetación; T2: pasto ovillo 'Currie', trébol subterráneo 'Campeda' y Antas', y trébol balansa 'Paradana'; T3: ballica anual 'Wimmera', trébol subterráneo 'Campeda' y 'Antas', y trébol balansa 'Paradana'; T4: falaris 'Holdfast', trébol subterráneo ‘Campeda' y 'Antas' y trébol balansa 'Paradana'; T5: festuca 'Manade', trébol subterráneo 'Campeda' y 'Antas', y trébol balansa 'Paradana'.

valores aumentan levemente, presentando en el tratamiento T5, con festuca y tréboles anuales, el mayor contenido (18,82\%), y en el tratamiento $\mathrm{T} 2$, con pasto ovillo y tréboles anuales, el menor valor $(16,66 \%)$. Esto puede ser explicado por el aumento de la retención, un menor requerimiento hídrico y una disminución de la evaporación del suelo. Al respecto, Celette et al. (2008) indicaron que la cobertura vegetal puede modificar espacial y temporalmente el régimen hídrico de un viñedo, concentrando raíces bajo la hilera del viñedo, modificando su enraizamiento y explorando zonas más profundas del suelo.

Respecto a las profundidades de $60-80$ y 80 $100 \mathrm{~cm}$, los valores de humedad aumentaron, siendo a los 60-80 cm (Fig. 4) el tratamiento T5, con festuca y leguminosas anuales, el de mayor contenido (19,23\%), y el tratamiento T2, con pasto ovillo y leguminosas anuales el de menor valor $(16,85 \%)$. Finalmente, la Fig. 5 muestra los valores a 80-100 $\mathrm{cm}$ de profundidad, observándose que el tratamiento T5, con festuca y tréboles anuales, muestra el mayor valor $(19,04 \%)$, mientras que el menor valor corresponde al tratamiento T4, con falaris, trébol subterráneo y trébol balansa $(17,11 \%)$. En ambas profundidades los contenidos de humedad son mayores por el aumento de la capacidad de retención de humedad del suelo y porque la profundidad de raíces del viñedo alcanza hasta $60 \mathrm{~cm}$.

En la entre hilera los valores de humedad determinados en esta investigación pueden estar relacionados con la morfología del sistema radical de las forrajeras, que alcanzan profundidades entre 20 a $30 \mathrm{~cm}$, con menor extracción de agua en profundidad, a excepción de falaris y festuca que poseen sistemas radicales fibrosos hasta $1 \mathrm{~m}$ de profundidad (Celette et al., 2005). Los resultados indican que entre tratamientos y profundidades no existieron diferencias estadísticas significativas en la cantidad de agua presente en el suelo en la entrehilera, no existiendo competencia por agua entre la cobertura vegetal y el viñedo.

Respecto del comportamiento de las especies en relación al contenido de agua, destacó la combinación festuca, trébol subterráneo y trébol balansa, ya que estas especies aumentan la cobertura del suelo sobre el 70\% (McGourty et al., 2008). Al respecto, la festuca es una especie recomendable para viñedos de lomajes y suelos poco profundos, ya que contribuye con menos de $1 \mathrm{~mm} \mathrm{H}_{2} \mathrm{O} \mathrm{d}^{-1}$ en la evapotranspiración del viñedo, principal preocupación de los productores respecto del consumo de agua por la cobertura vegetal (Guerra y Steenwerth, 2012). Sin embargo, según Cellete et al. (2005) la interacciones 


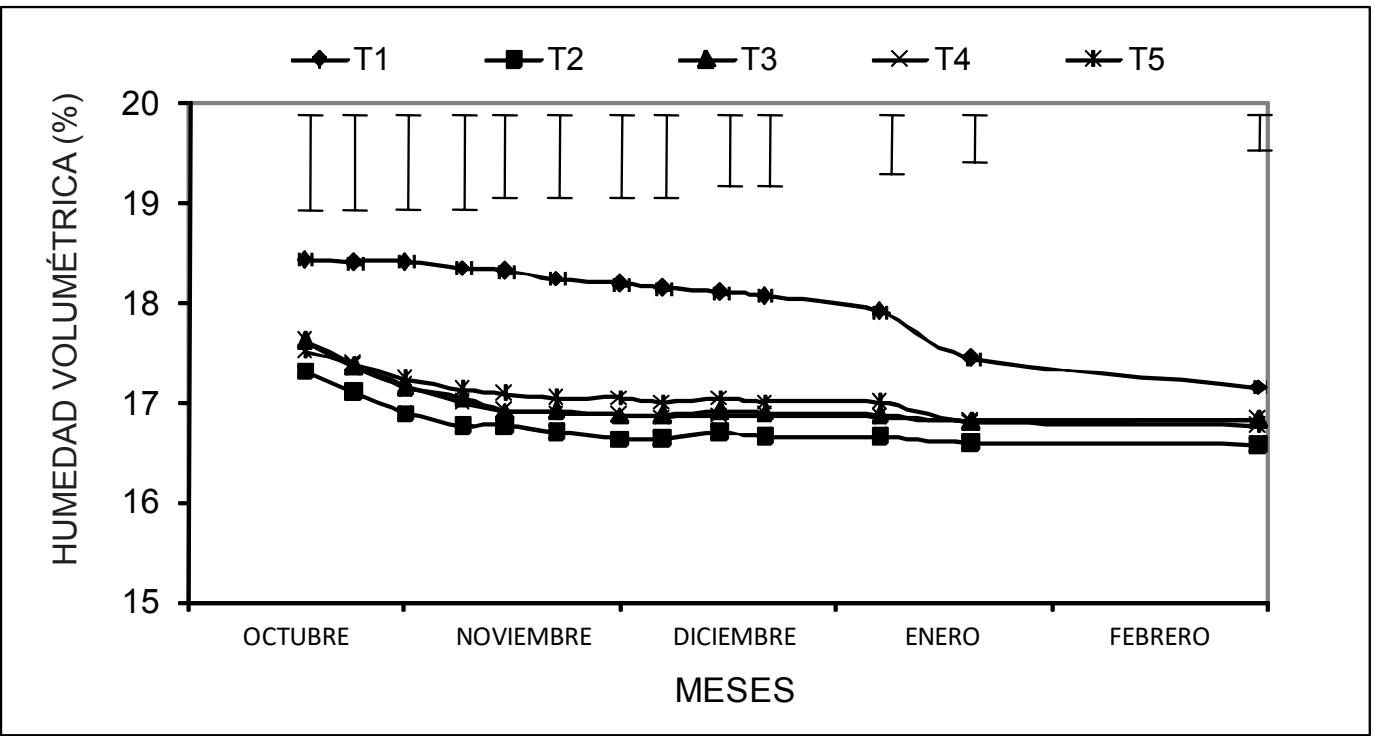

Fig. 2. Humedad volumétrica (\%) en un viñedo de secano Vitis vinifera 'Cabernet Sauvignon' en un suelo Typic Palexeralf a la profundidad de $20-40 \mathrm{~cm}$ bajo diferentes cubiertas vegetales entrehilera en la zona centro-sur de Chile.

Fig. 2. Soil volumetric soil water content (\%) at $20-40 \mathrm{~cm}$ depth in a non-irrigated vineyard Vitis vinifera 'Cabernet Sauvignon' under different inter-row cover crops in a Typic Palexeralf soil of central-southern Chile.

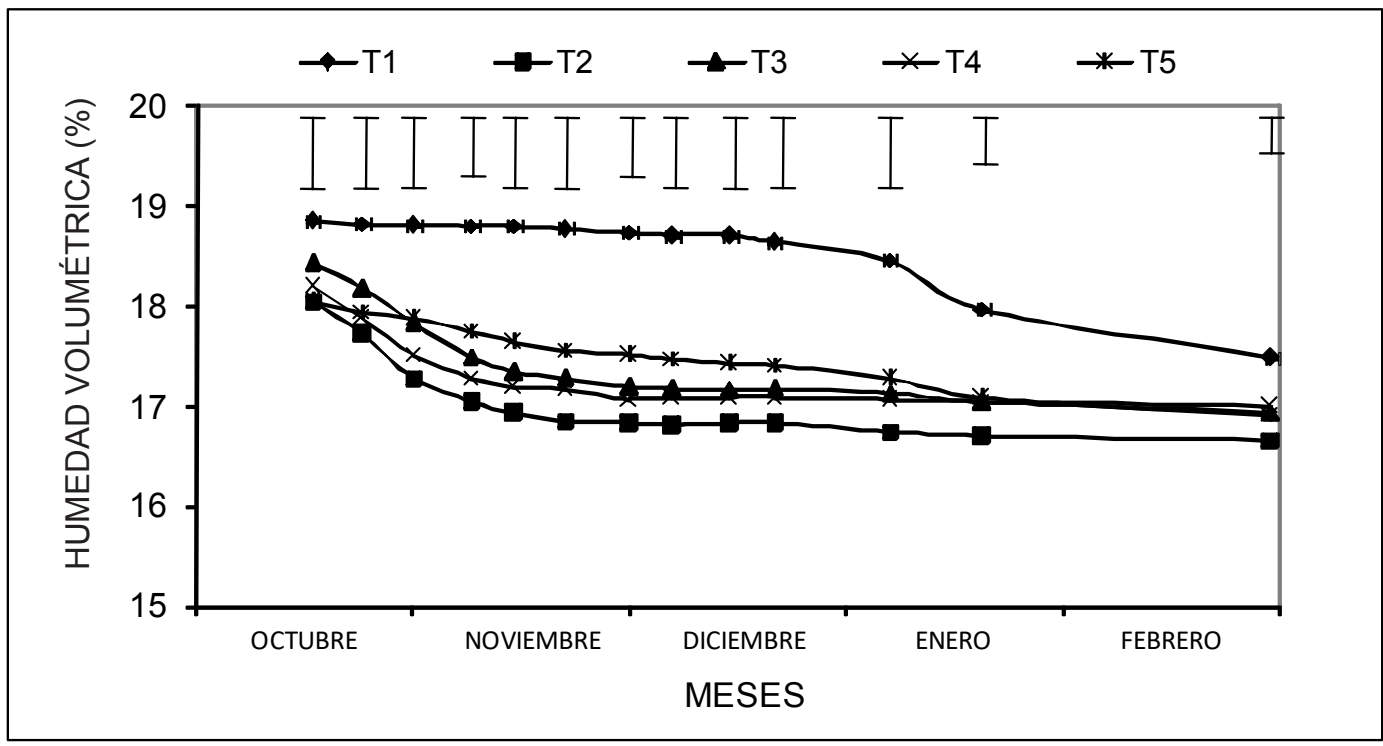

Fig. 3. Humedad volumétrica (\%) en un viñedo de secano Vitis vinifera 'Cabernet Sauvignon' en un suelo Typic Palexeralf a la profundidad de $40-60 \mathrm{~cm}$ bajo diferentes cubiertas vegetales entrehilera en la zona centro-sur de Chile.

Fig. 3. Volumetric soil water content (\%) at $40-60 \mathrm{~cm}$ depth in a non-irrigated vineyard Vitis vinifera 'Cabernet Sauvignon' under different inter-row cover crops in a Typic Palexeralf soil of central-southern Chile.

T1: testigo sin vegetación; T2: pasto ovillo 'Currie', trébol subterráneo 'Campeda' y 'Antas' y trébol balansa 'Paradana'; T3:ballica anual 'Wimmera', trébol subterráneo 'Campeda' y 'Antas' y trébol balansa 'Paradana'; T4: falaris 'Holdfast', trébol subterráneo cv. 'Campeda' y 'Antas' y trébol balansa 'Paradana'; T5: festuca 'Manade', trébol subterráneo cv. 'Campeda' y 'Antas' y trébol balansa 'Paradana'. 


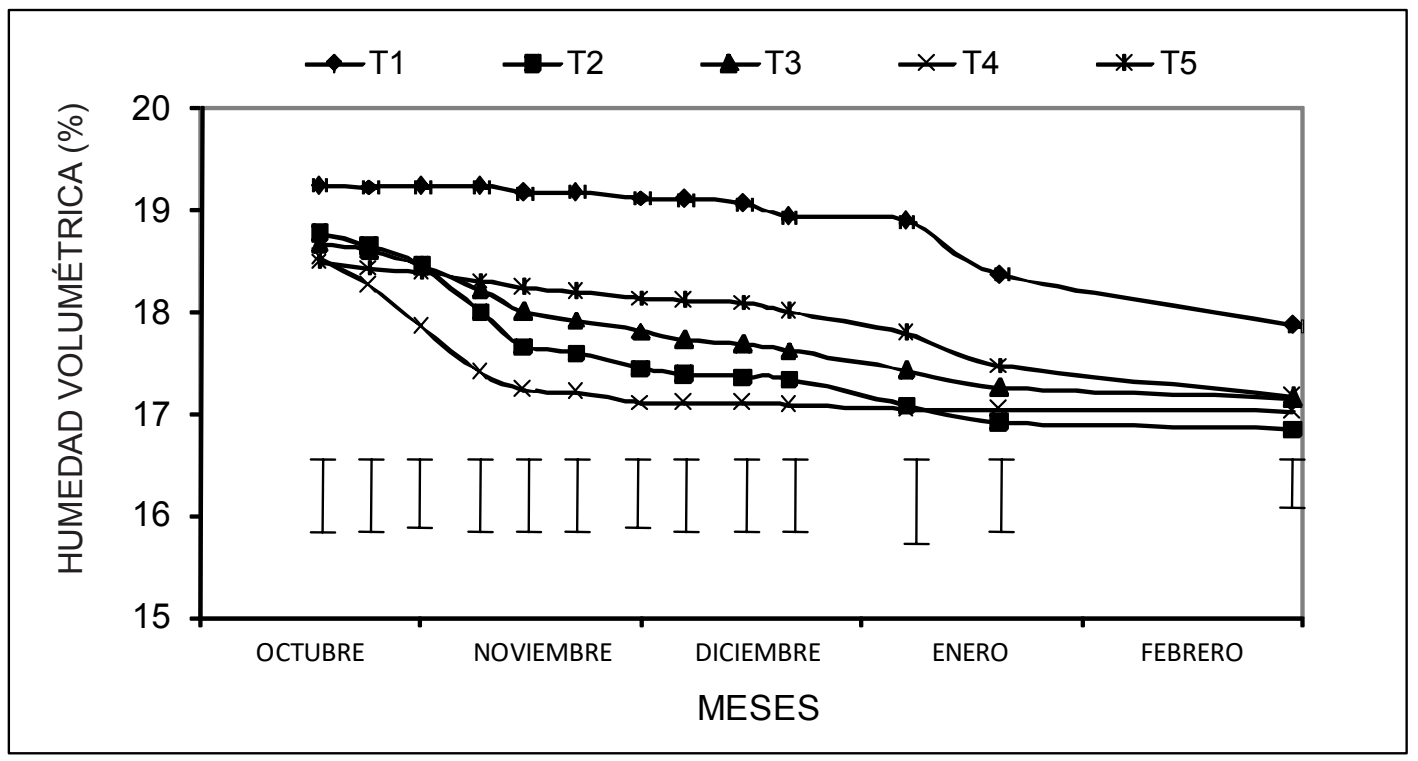

Fig. 4. Humedad volumétrica (\%) en un viñedo de secano Vitis vinifera 'Cabernet Sauvignon' en un suelo Typic Palexeralf a la profundidad de $60-80 \mathrm{~cm}$ bajo diferentes cubiertas vegetales entre hilera en la zona centro-sur de Chile.

Fig. 4. Soil volumetric water content $(\%)$ at $60-80 \mathrm{~cm}$ depth in a non-irrigated vineyard Vitis vinifera 'Cabernet Sauvignon' under different inter-row cover crops in a Typic Palexeralf soil of central-southern Chile.

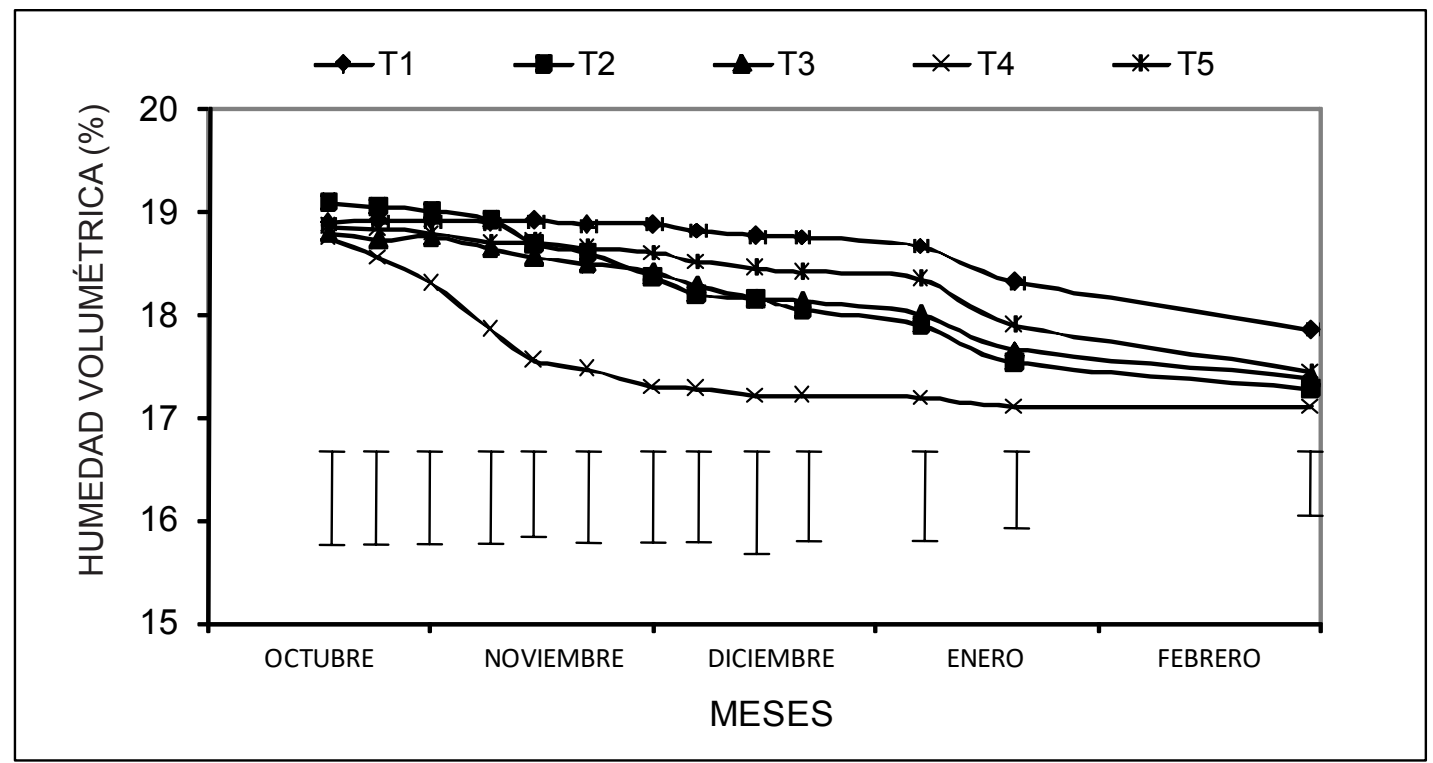

Fig. 5. Humedad volumétrica (\%) en un suelo Typic Palexeralf con Vitis vinifera 'Cabernet Sauvignon' a la profundidad de $80-100 \mathrm{~cm}$ con diferentes cubiertas vegetales entrehilera.

Fig. 5. Volumetric humidity (\%) at $80-100 \mathrm{~cm}$ depth in a Typic Palexeralf soil with Vitis vinifera 'Cabernet Sauvignon') under different inter-row cover crops.

T1: testigo sin vegetación; T2: pasto ovillo 'Currie', trébol subterráneo 'Campeda' y 'Antas' y trébol balansa 'Paradana'; T3:ballica anual 'Wimmera', trébol subterráneo 'Campeda' y 'Antas' y trébol balansa 'Paradana'; T4: falaris 'Holdfast', trébol subterráneo cv. 'Campeda' y 'Antas' y trébol balanza 'Paradana'; T5: festuca 'Manade', trébol subterráneo cv. 'Campeda' y 'Antas' y trébol balansa 'Paradana'. 
subterráneas entre la asociación herbáceaperenne, después de una investigación de 4 años en viña y festuca, aún no están muy claras, ya que el intercultivo disminuyó la cantidad de agua disponible y mejoró la infiltración del agua de lluvia invernal, probablemente al limitar el escurrimiento superficial. Además, interactuó con la vid y redujo su vigor vegetativo, por otros mecanismos distintos a la competencia por el agua, como nutrientes y alelopatía.

\section{Contenido volumétrico de agua en el suelo en la sobre hilera del viñedo}

El contenido de humedad volumétrica del suelo en la sobrehilera para todas las profundidades evaluadas (Tabla 2) no presentó diferencias

Tabla 2. Contenido de humedad volumétrica (\%) mensual a diferentes profundidades en la sobrehilera de un viñedo de secano 'Cabernet Sauvignon' en un suelo Typic Palexeralf en la zona centro sur de Chile, durante la temporada 2007-2008.

Table 2. Monthly volumetric water content (\%) at different depths on the row of a non-irrigated 'Cabernet Sauvignon' vineyard in a Typic Palexeralf soil of central-southern Chile, during the 2007-2008 season.

\begin{tabular}{|c|c|c|c|c|c|}
\hline \multirow{2}{*}{$\begin{array}{l}\text { Prof. / Mes } \\
0-20 \mathrm{~cm}\end{array}$} & \multicolumn{5}{|c|}{ Tratamientos } \\
\hline & $\mathrm{T} 1$ & $\mathrm{~T} 2$ & T3 & $\mathrm{T} 4$ & T5 \\
\hline Octubre & 16,38 & 16,70 & 16,55 & 16,51 & 16,84 \\
\hline Noviembre & 16,13 & 16,36 & 16,27 & 16,19 & 16,44 \\
\hline Diciembre & 16,03 & 16,23 & 16,17 & 16,19 & 16,26 \\
\hline Enero & 15,75 & 15,91 & 15,91 & 15,82 & 15,94 \\
\hline Febrero & 15,69 & 15,83 & 15,82 & 15,86 & 15,83 \\
\hline Promedio & 16,00 & 16,20 & 16,14 & 16,11 & 16,26 \\
\hline $20-40 \mathrm{~cm}$ & $\mathrm{~T} 1$ & $\mathrm{~T} 2$ & T3 & $\mathrm{T} 4$ & T5 \\
\hline Octubre & 18,28 & 18,09 & 18,45 & 17,85 & 18,16 \\
\hline Noviembre & 18,07 & 17,92 & 18,26 & 17,67 & 17,93 \\
\hline Diciembre & 17,80 & 17,69 & 17,97 & 17,50 & 17,62 \\
\hline Enero & 17,23 & 17,27 & 17,52 & 17,09 & 17,18 \\
\hline Febrero & 16,96 & 16,88 & 17,13 & 16,83 & 16,79 \\
\hline Promedio & 17,67 & 17,57 & 17,87 & 17,39 & 17,54 \\
\hline $40-60$ & $\mathrm{~T} 1$ & $\mathrm{~T} 2$ & $\mathrm{~T} 3$ & $\mathrm{~T} 4$ & $\mathrm{~T} 5$ \\
\hline Octubre & 18,57 & 18,53 & 18,76 & 18,18 & 18,12 \\
\hline Noviembre & 18,42 & 18,41 & 18,63 & 18,04 & 17,96 \\
\hline Diciembre & 18,27 & 18,24 & 18,47 & 17,85 & 17,82 \\
\hline Enero & 17,75 & 17,65 & 17,91 & 17,49 & 17,41 \\
\hline Febrero & 17,15 & 17,14 & 17,27 & 16,97 & 16,87 \\
\hline Promedio & 18,03 & 17,99 & 18,21 & 17,71 & 17,64 \\
\hline $60-80$ & $\mathrm{~T} 1$ & $\mathrm{~T} 2$ & $\mathrm{~T} 3$ & $\mathrm{~T} 4$ & T5 \\
\hline Octubre & 18,80 & 18,83 & 18,81 & 18,28 & 18,17 \\
\hline Noviembre & 18,74 & 18,74 & 18,71 & 18,17 & 18,14 \\
\hline Diciembre & 18,65 & 18,56 & 18,55 & 18,04 & 18,09 \\
\hline Enero & 18,16 & 17,99 & 18,03 & 17,62 & 17,71 \\
\hline Febrero & 17,37 & 17,39 & 17,29 & 17,02 & 17,04 \\
\hline Promedio & 18,34 & 18,30 & 18,28 & 17,83 & 17,83 \\
\hline $80-100$ & $\mathrm{~T} 1$ & $\mathrm{~T} 2$ & $\mathrm{~T} 3$ & $\mathrm{~T} 4$ & $\mathrm{~T} 5$ \\
\hline Octubre & 18,75 & 18,85 & 18,82 & 18,43 & 18,49 \\
\hline Noviembre & 18,70 & 18,76 & 18,81 & 18,35 & 18,42 \\
\hline Diciembre & 18,60 & 18,63 & 18,67 & 18,37 & 18,30 \\
\hline Enero & 18,24 & 18,24 & 18,12 & 17,84 & 18,01 \\
\hline Febrero & 17,49 & 17,52 & 17,33 & 17,18 & 17,24 \\
\hline Promedio & 18,36 & 18,40 & 18,35 & 18,03 & 18,09 \\
\hline
\end{tabular}

T1: testigo sin vegetación; T2: pasto ovillo 'Currie', trébol subterráneo 'Campeda' y 'Antas' y trébol balansa 'Paradana'; T3:ballica anual 'Wimmera', trébol subterráneo 'Campeda' y 'Antas' y trébol balansa 'Paradana'; T4: falaris 'Holdfast', trébol subterráneo 'Campeda' y 'Antas' y trébol balansa 'Paradana'; T5: festuca 'Manade', trébol subterráneo 'Campeda' y 'Antas' y trébol balansa 'Paradana'. 
significativas $(P>0,05)$ entre tratamientos. El contenido de humedad muestreado durante toda la temporada en forma semanal a diferentes profundidades, no fue afectado por las cubiertas vegetales, por lo cual se puede inferir que el intercultivo después de tres temporadas desde la siembra (Mayo 2005) no afectó la disponibilidad de agua de la planta en condición de secano. Los valores de humedad volumétrica empezaron a disminuir en los meses de mayor demanda evapotranspirativa en las profundidades de 0-20, 20-40, 40-60 cm, desde donde la planta extrae la mayor cantidad de agua, ya que las raíces se concentran en los primeros 60 $\mathrm{cm}$ de profundidad del suelo.

Los valores de contenido volumétrico de agua fluctuaron entre 15,69 a $16,84 \%$ en la profundidad de $0-20 \mathrm{~cm} ; 16,79$ a $18,45 \%$ en $20-40 \mathrm{~cm} ; 16,87$ a $18,76 \%$ en $40-60 \mathrm{~cm} ; 17,02$ a $18,83 \%$ en $60-80 \mathrm{~cm}$, y 17,18 a $18,85 \%$ en $80-100 \mathrm{~cm}$, no mostrando una tendencia definida en función de los tratamientos de cobertura vegetal. Esto permitiría inferir que no se presenta competencia entre la vid y cobertura vegetal por la disponibilidad de agua, aunque se requiere mayor investigación en esta asociación de plantas durante varias temporadas.

\section{Humedad aprovechable}

Los valores obtenidos para cada tratamiento de cobertura vegetal se presentan en la Tabla 3 , con promedios entre 6,99 y $8,41 \%$, donde el menor valor corresponde al testigo sin vegetación (T1) y el mayor a pasto ovillo con leguminosas anuales (T2). Estos valores son normales para este tipo de suelos, pero no pueden asociarse con efecto de las cubiertas vegetales, ya que sus efectos solo es posible determinarlos en ensayos de largo duración. La humedad aprovechable es baja, ya que en estos suelos graníticos destacan los altos contenidos de arena $(68,5 \%)$, y los bajos contenidos de arcilla $(11,8 \%)$, típicos de un suelo de la Serie Cauquenes, y las cubiertas vegetales no tendrían mayor efecto en este parámetro hídrico, pero ayudarían reducir el escurrimiento superficial y la erosión por lluvias, en comparación a una entrehilera sin vegetación. Los valores de la humedad aprovechable en función de la profundidad son bajos para todas las coberturas vegetales y profundidades de $0-5,5-10$ y $10-20$ $\mathrm{cm}$, donde el tratamiento $\mathrm{T} 2$ con pasto ovillo y tréboles anuales obtiene $8,41 \%$, y T5 con festuca y tréboles anuales un $8,28 \%$.

\section{Rendimiento}

El análisis de los parámetros de rendimiento (Tabla 4) indica que las cubiertas vegetales no presentaron diferencias significativas en peso de uva, peso de 10 bayas y peso de poda. No obstante lo anterior, el tratamiento testigo (T1) obtuvo el mejor rendimiento con 6.499 g planta $^{-1}\left(18,56\right.$ t ha $\left.^{-1}\right)$ en comparación al mejor tratamiento de cubiertas vegetales T5 de festuca y tréboles anuales con 5.893 g planta $^{-1}\left(16,84 \mathrm{t} \mathrm{ha}^{-1}\right)$ con una disminución del 9,32\% en el rendimiento. En cuanto a peso de bayas no se presentaron diferencias significativas entre tratamientos, aunque el mejor peso lo obtuvo el tratamiento T3 con ballica anual, trébol subterráneo y trébol balansa. En el peso de poda las diferencias son mayores con el testigo T1, con una disminución del 22,4\% en relación al T2 con pasto ovillo y leguminosas anuales, y también el intercultivo disminuyó el vigor del viñedo en los tratamientos con ballica (T3), falaris (T4) y festuca (T5), pero sin diferencias significativas. Al respecto, Pérez-Bermúdez et al. (2016) determinaron reducciones de rendimiento de 6,5\% con cubiertas vegetales, y Trigo-Córdoba et al. (2015) disminuciones del vigor sin comprometer rendimientos. Por su parte, Cellete et al. (2005) y Pou et al. (2011) en experimentos de larga duración reportaron disminución del contenido de agua para el cultivo y del rendimiento del viñedo. Estos diferentes resultados, según Pérez-

Tabla 3. Humedad aprovechable (\%) de un suelo Typic Palexeralf bajo un viñedo 'Cabernet Sauvignon' de secano con diferentes tratamientos de cubiertas vegetales en la entrehilera.

Table 3. Water availability (\%) in a Typic Palexeralf soil with a non-irrigated vineyard ‘Cabernet Sauvignon' under different inter-row cover crop treatments.

\begin{tabular}{lccccc}
\hline Profundidad (cm) & T1 & T2 & T3 & T4 & T5 \\
\hline $0-5$ & 7,32 & 8,17 & 7,70 & 7,02 & 7,98 \\
$5-10$ & 7,28 & 8,59 & 7,87 & 7,71 & 8,68 \\
$10-20$ & 6,37 & 8,47 & 7,74 & 6,35 & 8,17 \\
\hline Promedio & 6,99 & 8,41 & 7,77 & 7,03 & 8,28 \\
\hline
\end{tabular}

T1: testigo sin vegetación; T2: pasto ovillo 'Currie', trébol subterráneo 'Campeda' y 'Antas' y trébol balansa 'Paradana'; T3:ballica anual 'Wimmera', trébol subterráneo 'Campeda' y 'Antas'” y trébol balansa 'Paradana'; T4: falaris 'Holdfast', trébol subterráneo 'Campeda' y 'Antas' y trébol balanza 'Paradana'; T5: festuca 'Manade', trébol subterráneo 'Campeda' y 'Antas' y trébol balansa 'Paradana'. 
Tabla 4. Parámetros de rendimiento y madera de poda de un viñedo de secano 'Cabernet Sauvignon' sometido a diferentes tratamientos de cubiertas vegetales en la entrehilera.

Table 4. Yield components and pruning weight of a non-irrigated 'Cabernet Sauvignon' vineyard under different inter-row cover crop treatments.

\begin{tabular}{|c|c|c|c|}
\hline Tratamiento & $\begin{array}{l}\text { Peso uva } \\
\left(\text { g planta- }^{-1}\right)\end{array}$ & $\begin{array}{l}\text { Peso } 10 \text { bayas } \\
\text { (g) }\end{array}$ & $\begin{array}{l}\text { Peso poda } \\
\left.\text { (g planta }^{-1}\right)\end{array}$ \\
\hline $\mathrm{T} 1$ & $6.499 \mathrm{a}$ & $12,73 \mathrm{a}$ & $1.266 \mathrm{a}$ \\
\hline $\mathrm{T} 2$ & $5.439 \mathrm{a}$ & $12,40 \mathrm{a}$ & 982 a \\
\hline $\mathrm{T} 3$ & $5.593 \mathrm{a}$ & 13,13 a & $1.324 \mathrm{a}$ \\
\hline $\mathrm{T} 4$ & $5.800 \mathrm{a}$ & $12,94 \mathrm{a}$ & $1.023 \mathrm{a}$ \\
\hline $\mathrm{T} 5$ & $5.893 \mathrm{a}$ & $12,48 \mathrm{a}$ & $1.170 \mathrm{a}$ \\
\hline
\end{tabular}

T1: testigo sin vegetación; T2: pasto ovillo 'Currie', trébol subterráneo 'Campeda' y 'Antas' y trébol balansa 'Paradana'; T3:ballica anual 'Wimmera', trébol subterráneo 'Campeda' y 'Antas' y trébol balansa 'Paradana'; T4: falaris 'Holdfast', trébol subterráneo 'Campeda' y 'Antas' y trébol balanza 'Paradana'; T5: festuca 'Manade', trébol subterráneo 'Campeda' y 'Antas' y trébol balansa 'Paradana'.

a: sin diferencias significativas

Bermúdez et al. (2016) pueden estar asociados con el tipo de cubierta, clima, precipitación, nutrición, manejo del viñedo, y duración de los ensayos, pero conclusiones robustas solo se pueden lograr con experimentos de larga duración.

\section{CONCLUSIONES}

Las cubiertas vegetales establecidas en la entrehilera de un viñedo de secano 'Cabernet Sauvignon' en una zona mediterránea de la zona centro sur de Chile, no presentaron competencia por el recurso agua con las plantas del viñedo. El mejor tratamiento de intercultivo fue la combinación de festuca con trébol subterráneo y trébol balansa. El rendimiento y vigor de la vid no fueron afectados por el establecimiento de mezclas forrajeras en la entrehilera. Sin embargo se requieren experimento de larga duración para evaluar en forma más precisa la competencia entre cubiertas vegetales y el viñedo en zonas de secano.

\section{LITERATURA CITADA}

Balzarini, M.G., L. González, M. Tablada, F. Casanoves, J.A. Di Rienzo, y C.W. Robledo. 2008. InfoStat: software estadístico. Manual del usuario. Versión 2008. Brujas, Córdoba, Argentina.

Bouzas-Cid, Y., E. Trigo-Córdoba, I. Orriols, E. Falqué, and J.M. Mirás-Avalos. 2018. Influence of soil management on the red grapevine (Vitis vinifera L.) Mencía must amino acid composition and wine volatile and sensory profiles in a humid region. Beverages 4:76. doi: 10.3390/beverages4040076
Blanco-Canqui, H., T.M. Shaver J.L. Lindquist, C.A. Shapiro, R.W. Elmore, C.A. Francis, et al. 2015. Cover crops and ecosystem services: Insights from studies in temperate soils. Agronomy Journal 107(6):2449-2474.

Celette, F., J. Wery, E. Chantelot, J. Celette, and C. Gary. 2005. Belowground interactions in a vine (Vitis vinifera L.)-tall fescue (Festuca arundinacea Shreb.) intercropping system: water relations and growth. Plant Soil 276(12):205-217.

Celette, F., R. Gaudin, and C. Gary. 2008. Spatial and temporal changes to the water regime of a Mediterranean vineyard due to the adoption of cover cropping. Eur. J. Agron. 29(4):153-162.

Garcia, L., F. Celette, Ch. Garya, A. Ripoched, H. Valdés-Gómez, and A. Metay. 2018. Management of service crops for the provision of ecosystem services in vineyards: A review. Ecosystem \& Environment 251:118.

Guerra, B., and K. Steenwerth. 2012. Influence of floor management technique on grapevine growth, disease pressure, and juice and wine composition: A review. Am. J. Enol. Vitic. 63(2):149-164. doi:10.5344/ajev.2011.10001

Haruna, S.I., and N.V. Nkongolo. 2015. Cover crop management effects on soil physical and biological properties. Procedia Environ. Sci. 29:13-14.

Medrano, H., M. Tomás, D.S. Martorell, J.-M. Escalona, A, Pou, S. Fuentes, et al. 2015. Improving water use efficiency of vineyards in semi-arid regions. A review. Agron. Sustain. Dev. 35:499-517. 
McGourty, G., D. Tylicki, J. Nosrera, and A Toth. 2008. Self reeseeding anual legumes evaluated as cover crops for untilled vineyards. California Agricultura 62(54):191194.

Ovalle, C., A. del Pozo, A. Lavín, y J. Hirzel. 2007. Cubiertas vegetales en viñedos. Comportamiento de mezclas de leguminosas forrajeras anuales y efectos sobre la fertilidad del suelo. Agric. Técnica (Chile) 67(4):384392.

Pérez-Bermúdez, P., M. Olmo, J. Gil, L. GarcíaFérriz, C. Olmo, R. Boluda, et al. 2016. Cover crops and pruning in Bobal and Tempranillo vineyards have little influence on grapevine nutrition. Sci. Agric. 73(3):260-265.

Pou, A., J. Gulías, M. Moreno, M. Tomás, H. Medrano, and J. Cifre. 2011. Cover cropping in Vitis vinifera L. cv. Manto Negro vineyards under Mediterranean conditions: effects on plant vigour, yield and grape quality. J. Int. Sci. Vigne Vin 45(4):223-234.

Quezada, C., M. Sandoval, y A. Nario. 2010. Calibración de una sonda de neutrones en un suelo Haploxerands. Agro Sur 38(3):212-221.

Quezada, C., M.A. Soriano, J. Díaz, R. Merino, A. Chandía, J. Campos et al. 2014. Influence of soil physical properties on grapevine yield and maturity components in an Ultic Palexeralf soils, central-southern Chile. Open J. Soil Sci. 4(4):127-135.

Reyes, M., y P. Mejías. 2011. Caracterización de suelo y clima para la provincia de Cauquenes. P. 97-118. En M. Reyes y J. Díaz (eds.) Denominación de origen para el vino y aceite de oliva: una apuesta a la diferenciación de Cauquenes. Boletín INIA N ${ }^{\circ} 217$. Instituto de Investigaciones Agropecuarias, Villa Alegre, Chile

Ripoche, A., F. Celette, J.P. Cinna, and C. Gary. 2010. Design of intercrop management plans to fulfill production and environmental objectives in vineyards. Eur. J. Agron. 32:3039.
Ruiz, C. 2020. Vitivinicultura de Nuble: tradición agrícola para proyectar el futuro. p. 307328. En J. Gonzáez U. (ed) Agricultura de la nueva Región de Nuble: una caracterización sectorial. Libro $\mathrm{N}^{\circ}$ 39. INIA, Chillán, Chile.

Sandoval, M., J. Dörner, O. Seguel, J. Cuevas, y D. Rivera. 2012. Métodos de análisis físicos de suelos. Publicaciones del Departamento de Suelos y Recursos Naturales $N^{\circ} 5$. Universidad de Concepción, Facultad de Agronomía, Chillán, Chile.

Steenwerth, K.L., A.J. McElrone, A. CalderónOrellana, R.C. Hanifin, C. Storm, W. Collatz, et al. 2013. Cover crops and tillage in a mature Merlot vineyard show few effects on grapevines. Am. J. Enol. Vitic. 64(4):515-521.

Steenwerth, K.L., A. Calderón-Orellana, R.C. Hanifin, Ch. Storm, and A.J. McElrone. 2016. Effects of various vineyard floor management techniques on weed community shifts and grapevines water relations. Am. J. Enol. Vitic. 67(2):153-162.

Stolpe, N.B. 2006. Descripciones de los principales suelos de la VIII Región de Chile. Publicaciones del Departamento de Suelos y Recursos Naturales $N^{\circ} 1$. Universidad de Concepción,Facultad de Agronomía, Chillán, Chile.

Trigo-Córdoba, E., Y. Bouzas-Cid, I. OrriolsFernández, E. Díaz-Losada, and J.M. Mirás-Avalos. 2015. Influence of cover crop treatments on the performance of a vineyard in a humid region. Span. J. Agric. Res. 13(4):112.

Wheaton, A.D., B.M. Mckenzie, and J.M Tisdall. 2008. Management to increase the depth of soft soil improves soil conditions and grapevine performance in an irrigated vineyard. Soil Till. Res. 98(1):68-80. 